\title{
Developmental Changes in Medial Auditory Thalamic Contributions to Associative Motor Learning
}

\author{
Ka H. Ng and John H. Freeman \\ Department of Psychology, University of Iowa, Iowa City, Iowa 52242
}

Eyeblink conditioning (EBC) was used in the current study to examine the mechanisms underlying the ontogeny of associative motor learning in rats. Eyeblink conditioning emerges ontogenetically between postnatal day 17 (P17) and P24 in rats. Previous studies used electrical stimulation to show that the ontogeny of EBC is influenced by developmental changes in input from the medial auditory thalamus to the pontine nuclei, which in turn affects input to the cerebellum. The current study used tetrode recordings to examine the ontogeny of medial auditory thalamic sensory responses to the conditioned stimulus (CS) and learning-related activity during EBC. Rat pups were implanted with multiple tetrodes in the medial nucleus of the medial geniculate (MGm) and suprageniculate (SG) and trained on delay EBC on P17-P19, P24 -P26, or P31-P33 while recording spike activity. Developmental changes in MGm and SG sensory-related activity were found during a pretraining session with unpaired presentations of the auditory CS and periorbital stimulation unconditioned stimulus (US). Substantial developmental changes were observed in learning-related activity in the MGm and SG during CS-US paired training. The ontogenetic changes in learning-related activity may be related to developmental changes in input to the medial auditory thalamus from the amygdala and cerebellum. The findings suggest that the ontogeny of associative motor learning involves developmental changes in sensory input to the thalamus, amygdala input to the thalamus, thalamic input to the pontine nuclei, and cerebellar feedback to the thalamus.

\section{Introduction}

Eyeblink conditioning (EBC) is a commonly used method for examining associative motor learning (Freeman and Steinmetz, 2011). EBC involves repeated presentations of a conditioned stimulus (CS), typically a tone or light, followed by an unconditioned stimulus (US) that elicits eyelid closure. Auditory CS information is projected from the medial auditory thalamus (MAT) to the pontine nuclei and then to the cerebellum where it converges with US information emerging from the inferior olive via climbing fibers (Thompson and Steinmetz, 2009; Halverson et al., 2010; Freeman and Steinmetz, 2011). Repeated paired stimulation of the CS and US input pathways leads to the development of the eyeblink conditioned response (CR).

Eyeblink conditioning emerges ontogenetically between postnatal day 17 (P17) and P24 in rats (Stanton et al., 1992; Freeman, 2010). A developmental increase in sensory input to the pontine nuclei is a major factor in the emergence of auditory eyeblink conditioning. Electrical stimulation of pontine mossy fibers is an effective CS for eyeblink conditioning in rat pups as young as P12 (Campolattaro and Freeman, 2008), suggesting that the cere-

Received Jan. 19, 2012; revised March 10, 2012; accepted March 23, 2012.

Author contributions: J.H.F. designed research; K.H.N. performed research; K.H.N. analyzed data; K.H.N. and J.H.F. wrote the paper.

This work was supported by National Institutes of Health Grant NS038890 (J.H.F.). We thank Alireza Kashef and Matthew M. Campolattaro for technical assistance, Adam B. Steinmetz for statistical support, and Mary E. Goldsberry for editorial assistance.

Correspondence should be addressed to John H. Freeman, Department of Psychology, University of lowa, lowa City, IA 52242. E-mail: john-freeman@uiowa.edu.

DOI:10.1523/JNEUROSCI.0284-12.2012

Copyright $\odot 2012$ the authors $\quad 0270-6474 / 12 / 326841-10 \$ 15.00 / 0$ bellum is capable of learning early in ontogeny if mossy fiber stimulation is sufficiently strong. In contrast to mossy fiber stimulation, MAT stimulation as a CS does not result in early eyeblink conditioning (Freeman and Campolattaro, 2008). This finding suggests that there may be a developmental bottleneck in the MAT projection to the pons such that MAT neurons in younger pups receive a mature level of excitatory input from the inferior colliculus and cochlear nuclei but cannot adequately relay this auditory stimulation to the pontine nuclei (Campolattaro et al., 2007). The bottleneck hypothesis is further supported by neuronal recordings from the pontine nuclei, which show a developmental increase in the magnitude of activity elicited by a tone CS (Freeman and Muckler, 2003).

The current study examined developmental changes in MAT activity during EBC with multiple tetrodes to test the bottleneck hypothesis and to examine developmental changes in learningrelated activity. A prediction of the bottleneck hypothesis is that no developmental differences should be observed in MAT shortlatency sensory responses to an auditory CS before EBC. Shortlatency activity is elicited almost immediately after CS onset and is present before learning. Neurons in the MAT of adult rats also exhibit learning-related increases in the magnitude of shortlatency sensory activity and long-latency activity, which develops during EBC (Halverson et al., 2010). Long-latency activity is observed toward the end of the CS period and is correlated with CR production. These two types of learning-related neuronal activity may reflect different sources of input to the MAT; short-latency activity may be driven by the amygdala (Maren et al., 2001; Poremba and Gabriel, 2001; Halverson et al., 2010), whereas changes in long-latency activity may be driven by feedback from 


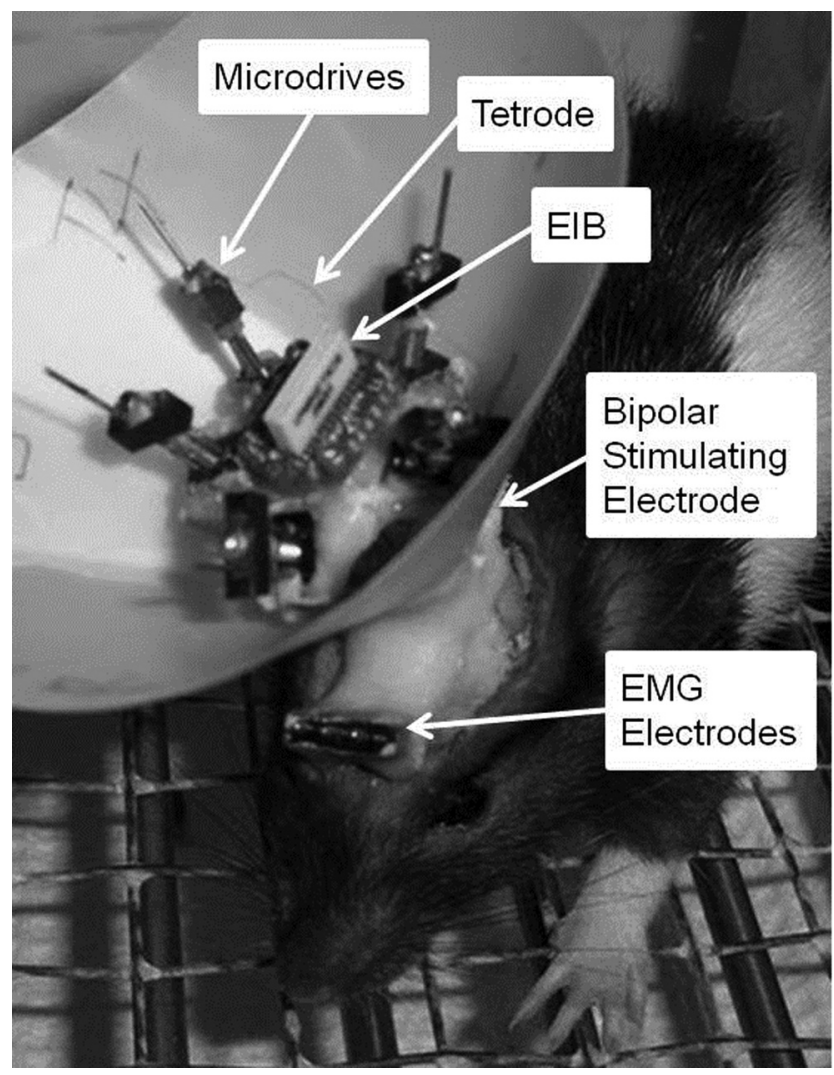

Figure 1. Image of a rat pup that depicts the eyeblink and neuronal recording equipment. Orbicularis oculi activity was recorded with EMG electrodes. The bipolar stimulating electrode delivered the periorbital stimulation unconditioned stimulus. Tetrodes were secured to the electronic interface board (EIB) and individually housed within microdrives. The recording head stage relayed unit activity from the EIB to the recording equipment.

the cerebellum (Halverson et al., 2010). The current study found developmental changes in learning-related MAT activity that paralleled developmental changes in behavioral learning.

\section{Materials and Methods}

Subjects. Subjects were 21 male and female Long-Evans rat pups trained at three different ages, $\mathrm{P} 17-\mathrm{P} 19(n=7), \mathrm{P} 24-\mathrm{P} 26(n=8)$, and P31-P33 $(n=6)$, from 18 litters. All rats were given ad libitum access to food and water, and maintained on a $12 \mathrm{~h}$ light/dark cycle. Rat pups trained on P24-P26 or P31-P33 were weaned on P19 and then housed individually following surgery. Rat pups trained on P17-P19 were returned to the litter and dam following surgery and between training sessions. The tetrode assembly was protected from the dam and littermates by a protective cap made of dental cement.

Surgery. Rat pups were anesthetized with isoflurane (1-5\%) $2 \mathrm{~d}$ before the start of training. Stainless-steel electromyography (EMG) electrodes for monitoring eyelid movement were implanted into the upper left orbicularis oculi muscle, and the EMG ground was secured to the skull slightly anterior to bregma. The EMG electrodes and ground wire terminated in gold pins in a plastic connector strip (Fig. 1). A bipolar stimulating electrode for delivering the US was implanted subdermally, caudal to the left eye (Fig. 1). Previous studies found that the contralateral MAT is necessary for eyeblink conditioning (Halverson and Freeman, 2006). A craniotomy for tetrodes was therefore performed above the right MAT with stereotaxic coordinates of $2.5 \mathrm{~mm}$ posterior and $2.7 \mathrm{~mm}$ lateral to lambda. Upon removal of the dura matter, tetrode bundles of the recording drive were implanted onto the brain surface, and the ground of the recording drive was secured to the skull slightly anterior to lambda. The remaining space between the drive and skull was sealed with low viscosity silicon (Kwik-Sil; World Precision Instruments). Bone cement (Zimmer) was use to secure the EMG electrodes, bipolar electrode, and the recording drive to the skull. Immediately after surgery, all of the recording tetrodes and the reference tetrode were lowered $2.24 \mathrm{~mm}$ into the brain. On the day after surgery, while the spike activity was monitored, the recording tetrodes were lowered again into either the suprageniculate (SG) or the medial nucleus of the medial geniculate (MGm).

Spike recording apparatus. The recording drive was composed of two or four recording tetrodes and a reference tetrode (Fig. 1). Each individual tetrode was made of four insulated nichrome wires $(12 \mu \mathrm{m}$ diameter; Kanthal) with one end twisted and partially heat-fused together and the other end fed into an electronic interface board (Neuralynx). Tetrode impedance was adjusted to $500 \mathrm{k} \Omega$ (impedance tester IMP-1; Bak Electronics) by sending a small current through each channel of the tetrode in gold plating solution (SIFCO Process). Once implanted, the depth for each tetrode could be reliably controlled by turning of a corresponding hex screw.

The spike signal was passed through the head stage and preamplified at unity gain (Neuralynx). The output was then amplified between 20,000 and $25,000 \times$, and bandpass filtered $(0.6-6.0 \mathrm{kHz}$; Neuralynx). Neural signals that passed an amplitude threshold were digitized and stored at 32 $\mathrm{kHz}$ (Cheetah System; Neuralynx).

Conditioning apparatus. The conditioning chamber (BRS/LVE) has a metal grid floor and was contained within a sound attenuation chamber. The CS was delivered through a speaker on one side of the conditioning chamber. The US was delivered by a stimulus isolator (model number 365A; World Precision Instruments). Presentation of the stimuli and recording of the eyelid EMG activity was controlled by computer software (JSA Designs). Differential EMG activity was filtered (500-5000 $\mathrm{Hz}$ ), amplified (2000×), and integrated (JSA Designs).

Conditioning procedure. Starting on the second day after surgery, the pups received two sessions of training per day for $3 \mathrm{~d}$. The first session was a pretraining session that included unpaired presentations of the CS and US. The other five sessions included paired CS-US trials. Training sessions were separated by at least $4 \mathrm{~h}$. The CS was a $2.0 \mathrm{kHz}, 82 \mathrm{~dB}$ tone that lasted for $400 \mathrm{~ms}$. During paired training, the US was a $25 \mathrm{~ms}$ periorbital shock (2-3 mA) that immediately followed the CS. The US intensity was adjusted in each rat to produce a slight head jerk. There were no age-related statistical differences in the US intensity. During unpaired training, the US was reduced to $1 \mathrm{~ms}$ to minimize the stimulation artifact in the neuronal recordings. Each unpaired session had a total of 200 trials with $100 \mathrm{CS}$-alone trials and 100 US-alone trials with an intertrial averaged $15.6 \mathrm{~s}$ (range, 10-22 s). Each paired session had a total of 100 trials, with 90 standard paired trials and 10 CS-alone trials. During paired trials, presentation of the $400 \mathrm{~ms}$ CS was followed immediately by the $25 \mathrm{~ms}$ US. During CS-alone trials, the CS was presented without the US. The intertrial interval for paired sessions averaged $30 \mathrm{~s}$ (range, 20-40 s). EMG signals that were 0.4 units above baseline activity during the CS, but not within the $80 \mathrm{~ms}$ startle period, were counted as a CR. The amplitude, onset latency, and peak latency of the CR were measured from CRs that occurred on CS-alone trials to avoid the US and UR contamination.

Unit separation. Cluster analysis was conducted to isolate single-unit activity from the multiunit recording. An automatic cluster separation program (Klusta-Kwik; K. Harris, Rutgers University, Newark, NJ) was used initially to separate units based on features of their waveforms including peak, valley, energy, and principal components. Each cluster was then further refined or combined with other clusters based on the same features (MClust-3.5; A. D. Redish, University of Minnesota, Minneapolis, MN). Waveform parameters, interspike intervals, autocorrelograms, overall firing rate, and stability were evaluated for each unit.

Spike analysis. Units were initially classified into different responses types. The $400 \mathrm{~ms}$ CS period was divided into four $100 \mathrm{~ms}$ intervals with $10 \mathrm{~ms}$ bins. $Z$-score analysis (normalized to the baseline) was performed on the peristimulus histograms for all units. The activity in each CS interval was compared against the baseline of the same unit ( $\alpha$ was adjusted for multiple comparisons to 0.01 ; all other statistical tests used an $\alpha$ of 0.05 ). Units with significantly increased activity only during the first and/or second intervals during the CS period were classified as shortlatency units. Units with significantly increased activity only during the third and/or forth intervals during the CS period were classified as longlatency units. Units that fit into both categories were classified as sus- 
A



B



Figure 2. Images of thionin-stained coronal sections showing locations of tetrode tips in the medial nucleus of the medial geniculate (MGm) and suprageniculate nucleus (SG) of the medial auditory thalamus. $A$, Tetrode tip within the MGm, lateral to the anterior pretectal nucleus (APN) and medial to the dorsal (MGd) and ventral (MGv) nuclei of the medial geniculate. $\boldsymbol{B}$, Tetrode tip in the SG.
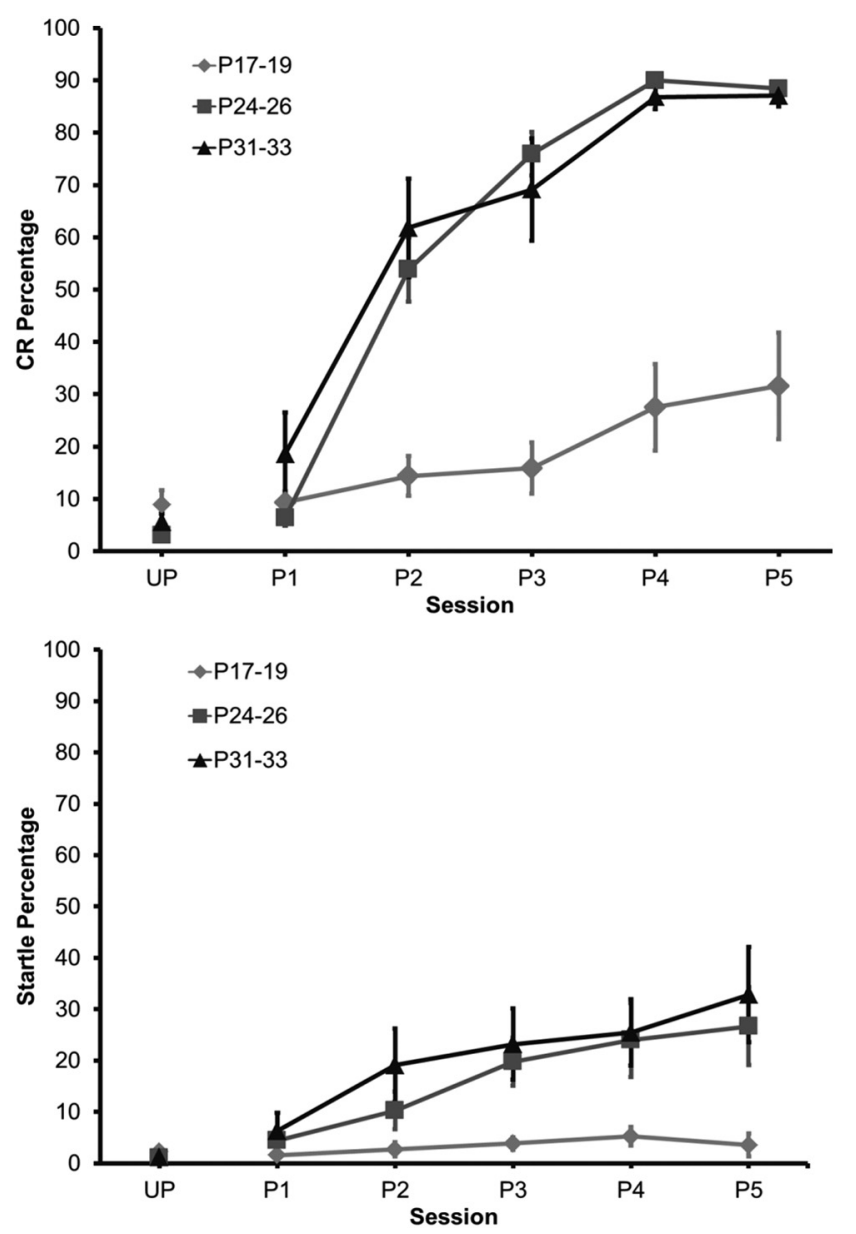

Figure 3. Conditioned response and startle response acquisition as a function of postnatal age. Mean (+SEM) eyeblink CR percentage (top) and startle percentage (bottom) during unpaired pretraining and five paired training sessions in rat pups trained on P17-P19, P24-P26, or P31-P33.

tained units. $\chi^{2}$ tests were performed for the percentage of unit response types as a function of age for both unpaired and paired training. For comparisons with small sample sizes, when the $\chi^{2}$ test is not suitable, Fisher's exact test was performed. For learning-related activity, the Wilcoxon signed-rank test was used to compare normalized activity on trials

with CRs versus trials without CRs. Population analyses were conducted using repeatedmeasures ANOVA on normalized activity.

Histology. After completion of the experiment, a small lesion was made to mark the tip of each tetrode with $8 \mathrm{~s}$ of $10 \mu \mathrm{A}$ current through one of the tetrode channels. The rats were anesthetized with sodium pentobarbital the next day, followed by perfusion with saline and $10 \%$ buffered formalin. The brains were then removed and placed in 30\% sucroseformalin. Brains were sectioned at $50 \mu \mathrm{m}$, mounted on glass slides, and stained with thionin. Tetrode placement was examined under a light microscope with a stereotaxic brain atlas (Swanson, 2004).

\section{Results}

\section{Tetrode placement}

A total of 786 neurons from 21 animals were analyzed. Of the 424 neurons recorded during the unpaired session, 204 were recorded from the MGm (P17-P19, 63; P24-P26, 97; P31$\mathrm{P} 33,44)$ and 220 were recorded from the SG (P17-P19, 84; P24$\mathrm{P} 26,68 ; \mathrm{P} 31-\mathrm{P} 33,68)$. Of the 362 neurons recorded during the last paired session, 189 were recorded form the MGm (P17-P19, 80; P24-P26, 71; P31-P33, 38) and 173 were recorded from the SG (P17-P19, 57; P24-P26, 51; P31-P33, 65). Examples of MGm and SG tetrode placements are displayed in Figure 2. On the day after surgery, small depth adjustments were made to the reference tetrode before recording to minimize background activity. The reference tetrodes were located in the dorsal hippocampal commissure $(n=9)$, dorsal subiculum $(n=4)$, corpus callosum $(n=$ $4)$, ventricle $(n=3)$, and stratum radiatum of CA1 $(n=1)$.

\section{Behavioral data}

The rate and magnitude of eyeblink conditioning were significantly lower in the youngest age group when compared with the older groups. The P24-P26 and P31-P33 groups, however, did not differ from one another. This age-related increase in conditioning was clearly evident across training sessions, after the initial unpaired session (Fig. 3). A repeated-measures ANOVA on the CR percentage data revealed a significant Age by Session interaction $\left(F_{(10,85)}=19.89 ; p<0.01\right)$, which was further examined with Tukey's honestly significant difference tests. There were no significant differences in the percentage of CRs between the age groups during the unpaired session. The percentage of CRs was higher for both P24-P26 and P31-P33 groups compared with the P17-P19 group from the second paired session to the last paired session, but there was no significant difference between the P24-P26 and P31-P33 groups.

Eyelid startle amplitude did not change during training, and there were no age-related differences in startle amplitude. The percentage of startle responses did not differ between age groups during unpaired training. However, there was an increase in startle frequency as training progressed, which also increased as a function of age (Fig. 3). A repeated-measures ANOVA revealed a significant Age by Session interaction $\left(F_{(10,85)}=3.08 ; p<0.003\right)$, which was due to a higher percentage of startle responses in the groups trained on $\mathrm{P} 24-\mathrm{P} 26$ and $\mathrm{P} 31-\mathrm{P} 33$ relative to the group trained on P17-P19.

\section{Neuronal activity during CS-US unpaired pretraining}

Neuronal activity recorded during the unpaired pretraining session provided a means for assessing developmental changes in the 
sensory responses of MAT neurons before learning. The proportion of thalamic neurons with significant changes in activity during the tone CS did not differ between the age groups for the MGm or SG during pretraining (Fig. 4).

Age-related differences in the magnitude of the population neuronal responses to the CS were analyzed with repeatedmeasures ANOVA of the normalized activity. In the MGm, there was a significant Age by Bin interaction $\left(F_{(78,5109)}=3.13 ; p<\right.$ $0.01)$. Post hoc tests revealed that the P24-P26 and P31-P33 age groups had significantly higher activity during the CS than the P17-P19 group in the first $60 \mathrm{~ms}$ (bins 1-6) of the CS (Figs. 5, 6). Although neuronal activity in the P24-P26 and P31-P33 age groups did not differ significantly during the early portion of the CS (0-40 ms, bins $1-4)$, activity in the P31-P33 group was significantly higher than activity in the P24-P26 group from 40 to 90 ms (bins 5-9) after the CS onset (Fig. 5). Thus, MGm neurons showed an age-related increase in both the magnitude and duration of activity during the CS.

In the $S G$, there was a significant Age by Bin interaction $\left(F_{(78,4914)}=5.38\right.$; $p<$ $0.01)$. Post hoc tests revealed that SG activity in the P17-P19 group was significantly higher than activity in the two older age groups for the first $20 \mathrm{~ms}$ (bins 1-2) of the CS (Figs. 5, 7). Although activity of P31P33 group had a longer peak latency, its amplitude was significantly higher than both the P17-P19 and P24-P26 groups from 30 to $100 \mathrm{~ms}$ (bins 4-10) after CS onset.

Developmental changes in neuronal activity within the SG and MGm were seen in the magnitude, duration, and latency of the population response but not in the overall percentage of neurons that responded to the tone CS. The developmental trends in the MGm and SG differed substantially, with an age-related increase in MGm activity but an age-related decrease in SG activity. Duration of the neuronal response to the CS increased with age in the MGm. In the SG, the latency of the peak of activity following CS onset became later with age. This complex pattern of developmental changes in MGm and SG activity was then compared with developmental changes in activity that emerged during paired CS-US training.

\section{Neuronal activity during CS-US paired training}

During the last paired CS-US training session, there was a significantly lower percentage of CS-responsive neurons for the P17$\mathrm{P} 19$ group relative to the $\mathrm{P} 24-\mathrm{P} 26$ and $\mathrm{P} 31-\mathrm{P} 33$ groups in the MGm and SG $(p<0.05)$. Among the responsive neurons in the $M G m$, there was a significantly higher percentage of neurons that showed short-latency responses for the $\mathrm{P} 17-\mathrm{P} 19$ group relative to the other groups $(p<0.01)$. In contrast, the P17-P19 group had a lower percentage of neurons with sustained activity $(p<0.01)$. This pattern of developmental differences in the MGm indicates that there was a shift from short-latency to sustained responses in the P24-P26 and P31-P33 groups but not in the P17-P19 group during CS-US training relative to unpaired pretraining. In the SG, no significant differences were found in the percentage of neurons with different activity profiles between the age groups.

In addition to the developmental shift from short-latency to sustained activity profiles, neurons in the MGm showed a clear age-related increase in the magnitude of activity during CS-US
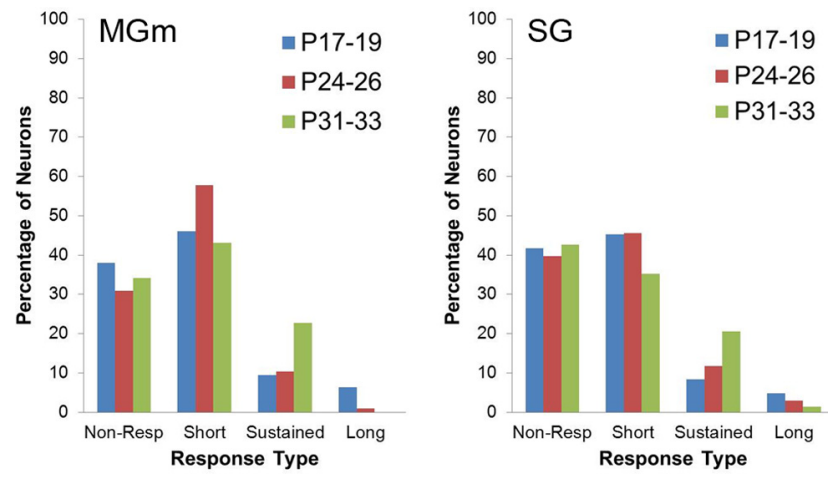

Figure 4. Percentage of neurons in the MGm and SG that were nonresponsive or exhibited significant increases in activity during unpaired pretraining. Neurons with significantly increased activity during only the first and/or second $200 \mathrm{~ms}$ intervals of the $400 \mathrm{~ms}$ tone conditioned stimulus period were classified as short latency. Neurons with significantly increased activity only during the third and/or forth $100 \mathrm{~ms}$ intervals were classified as long latency. Neurons that fit into both categories were classified as sustained.
Unpaired MGm

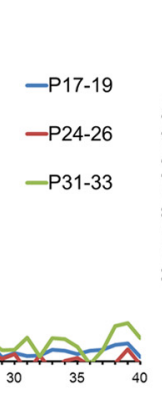

Unpaired SG

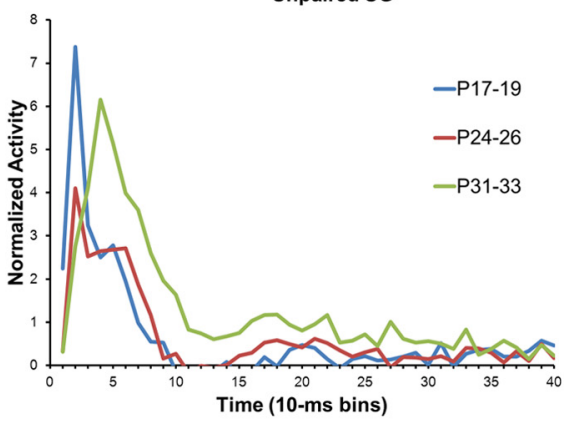



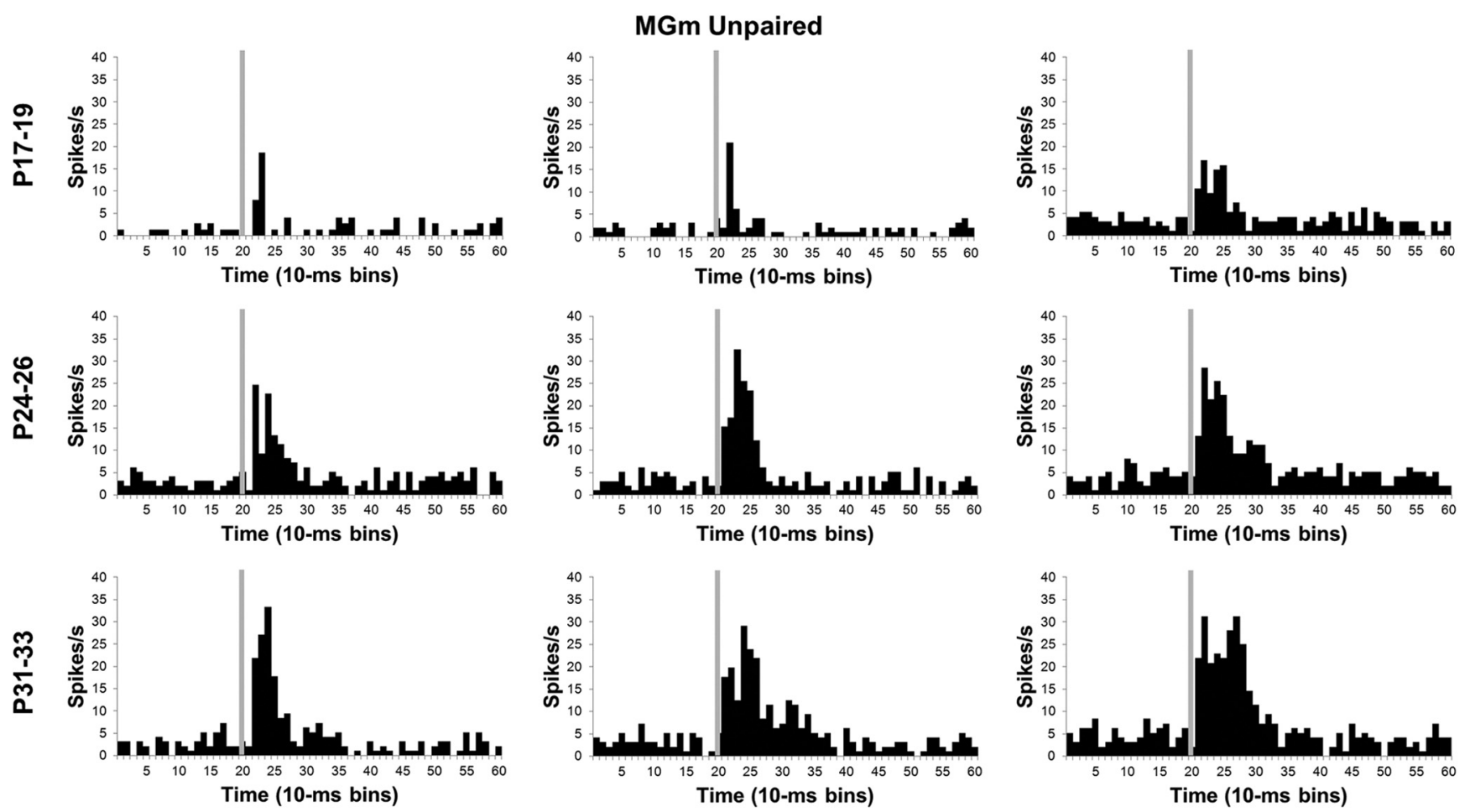

Figure 6. Histograms of single-neuron activity in the medial nucleus of the MGm during presentations of the $400 \mathrm{~ms}$ tone conditioned stimulus in the unpaired pretraining session. Three examples are displayed for the groups trained on P17-P19 (top row), P24-P26 (middle row), or P31-P33 (bottom row). Stimulus-elicited activity is greater in the neurons recorded from the older groups relative to the neurons recorded from the P17-P19 group during the first $100 \mathrm{~ms}$ of the tone stimulus. Stimulus onset is depicted by the vertical gray line.
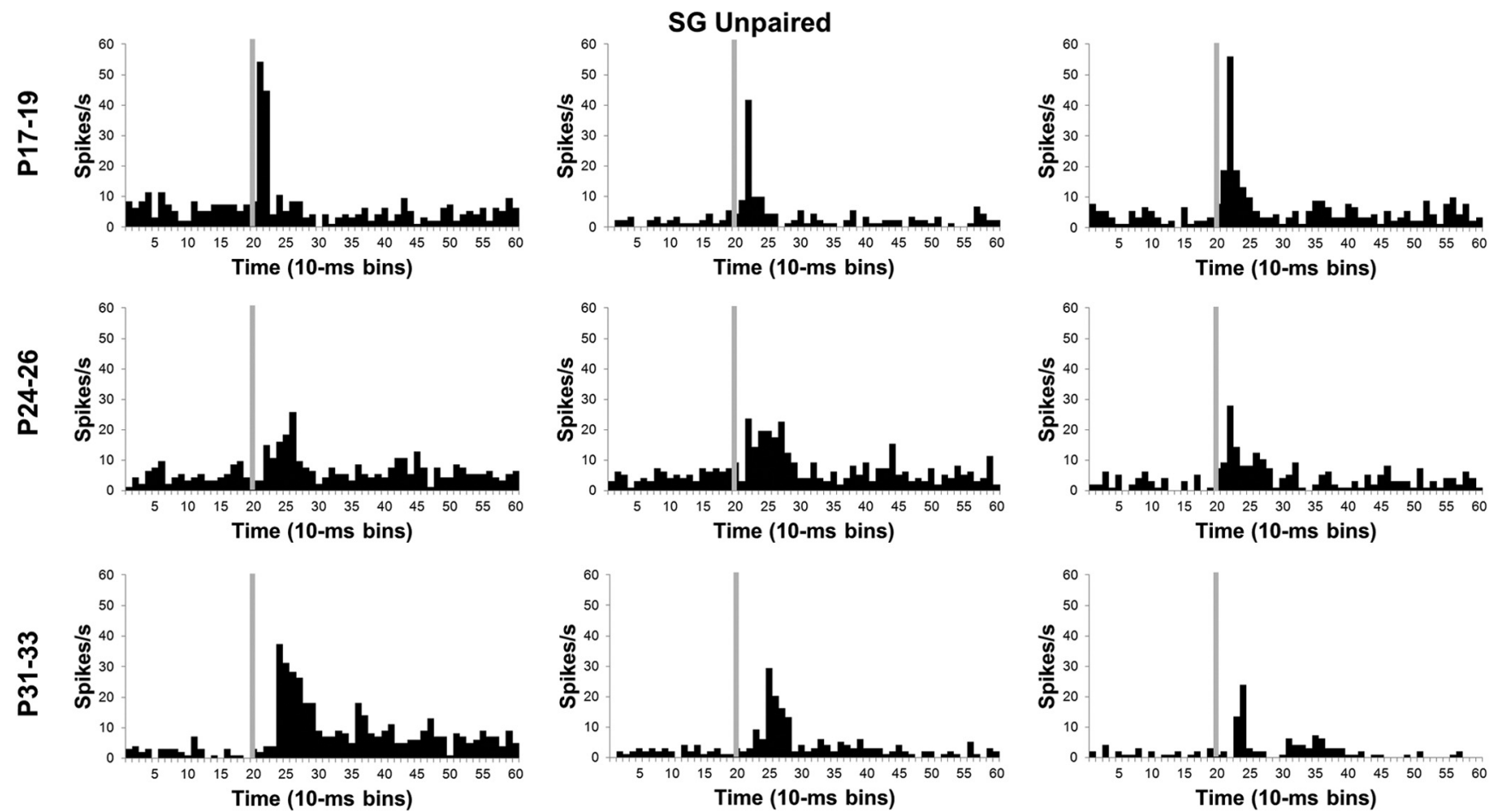

Figure 7. Histograms of single-neuron activity in the $\mathrm{SG}$ nucleus during presentations of the $400 \mathrm{~ms}$ tone conditioned stimulus in the unpaired pretraining session. Three examples are displayed for the groups trained on P17-P19 (top row), P24-P26 (middle row), or P31-P33 (bottom row). Stimulus-elicited activity is greater in the neurons recorded from the P17-P19 group relative to the neurons recorded from the older groups during the first $50 \mathrm{~ms}$ of the tone stimulus. Stimulus onset is depicted by the vertical gray line.

increases in activity only in the older groups; the P17-P19 neurons seem to actually show less activity during CS-US training relative to CS-US pretraining (see below, Comparison of neuronal activity between CS-US unpaired and CS-US paired training).
Learning-specific neuronal activity

A method used previously for assessing changes in neuronal activity that are specific to learning is to compare activity during trials with CRs and trials without CRs (Halverson et al., 2010; 
Campolattaro et al., 2011). A previous report examining MAT tetrode recordings in adult rats interpreted differential activity for CR and no-CR trials as reflecting learned attention to the CS driven by excitatory input from the amygdala and cerebellum (Halverson et al., 2010). In the current study, the activity of MAT neurons was compared between trials with CRs and trials without CRs in the three age groups to examine developmental changes in learned attention to the CS (Fig. 11). This analysis was conducted using data from the last paired session to ensure that all pups, including pups trained on P17-P19, produced enough CRs for valid comparisons.

In the MGm and SG of the youngest age group, there was no significant difference in the percentage of neurons that showed increased activity during CR trials compared with no-CR trials. However, a significantly higher percentage of neurons showed increased activity during trials with CRs relative to trials without CR in the MGm and SG for the P24-P26 and P31-P33 groups $(p<0.05)$.

An age-related increase in the magnitude of MGm activity was clearly evident on trials in which the rat produced a CR during CS-US paired training (Fig. 11). The differences in the magnitude of activity between CR and no-CR trials were examined using repeated-measures ANOVA for normalized activity. This analysis was conducted on the neurons that showed significantly increased activity during the CS. There was a significant Age by CR versus no-CR by Bin interaction $\left(F_{(78,4524)}=5.05 ; p<0.01\right)$. Post hoc test revealed that there was significantly more activity during trials with CRs than trials without CRs in the initial $70 \mathrm{~ms}$ (bins 1-7) after CS onset for the P31-P33 group and in the initial $60 \mathrm{~ms}$ (bins 1-6) after CS onset for the P24-P26 group. However, neurons in the P17-P19 group showed less activity in trials with CRs than trials without CRs in the initial $40 \mathrm{~ms}$ (bins 1-4) after CS onset. From 80 to $400 \mathrm{~ms}$ (bins 9-40) after CS onset, neurons in the P24-P26 and P31-P33 groups showed more activity on trials with CRs for 9 bins and 25 bins, respectively. In contrast, neurons in the P17-P19 group did not exhibit differential activity between 80 and $400 \mathrm{~ms}$ (bins 9-40) after CS onset. These findings indicate that the older groups showed greater learningrelated activity during the initial short-latency burst of activity following CS onset and later in the CS period, a pattern of results consistent with the adult recordings (Halverson et al., 2010). Moreover, the CR-related activity increased substantially from P24-P26 to P31-P33.

The general developmental trends for differences in activity during CR trials and no-CR trials observed in the MGm were also evident in the SG (Fig. 11). The P17-P19 group showed no differential activity during the initial period of the CS, whereas the P24-P26 and P31-P33 groups showed significantly elevated activity during $\mathrm{CR}$ trials relative to no-CR trials during the first 10-30 ms (bins 2-3). The ANOVA revealed an Age by CR versus no-CR by Bin interaction $\left(F_{(78,2652)}=1.29 ; p<0.05\right)$. Post hoc tests indicated that there was more activity during trials with CRs for 10-30 ms (bins 2-3) and 40-50 ms (bin 5) after CS onset for the P24-P46 group and 10-40 ms (bins 2-4) after CS onset for the P31-P33 group. The was also significantly more activity during trials with CRs 330-340 (bin 34) and 370-380 ms (bin 38) after CS onset for the P17-P19 group, and 380-390 ms (bin 39) after CS onset for the P31-P33 group.

\section{Comparison of neuronal activity between CS/US unpaired and CS-US paired training}

The response profiles of neurons in the MGm and SG shifted from primarily short-latency responses to sustained responses in the P24-P26 and P31-P33 groups as training switched from unpaired to paired training. In contrast, MGm and SG neurons in the P17-P19 group were primarily nonresponsive or showed short-latency responses during paired training.

The transition from unpaired to paired training resulted in increased MGm activity throughout the CS in the older age groups but not in the P17-P19 group (Fig. 12). The pairingspecific increases in activity were similar to those seen in adult rats in a previous study (Halverson et al., 2010). Repeatedmeasures ANOVA revealed significant Session by $\operatorname{Bin}\left(F_{(39,10,218)}=\right.$ $1.68 ; p<0.01)$ and Age by $\operatorname{Bin}\left(F_{(78,10,218)}=4.16 ; p<0.01\right)$ interactions. Post hoc tests indicated that there was significantly more activity during paired relative to unpaired training 20-40 $\mathrm{ms}$ (bins 3-4) after CS onset for the P24-P26 and P31-P33 age groups, but not for the P17-P19 group. Pairing-specific increases in activity were also seen later in the CS period for P24-P26 and P31-P33 groups, but not in the P17-P19 group.

In the SG, there was a developmental change in the difference between activity during unpaired and paired training (Fig. 12), which was indicated by an Age by Session by Bin interaction $\left(F_{(78,8892)}=2.51 ; p<0.01\right)$. The $\mathrm{P} 24-\mathrm{P} 26$ and $\mathrm{P} 31-\mathrm{P} 33$ groups showed greater activity during paired training 10-30 ms (bins $2-3)$ after CS onset $(p<0.01)$, whereas the P17-P19 group showed a decrease in short-latency activity during paired training relative to unpaired training $(p<0.05)$. In contrast to the $\mathrm{MGm}$, there were very minor increases in activity during paired training later in the CS period.

Overall, recordings in the MAT showed a developmental change in the magnitude of learning-related activity. The P24P26 and P31-P33 groups showed greater activity during paired training relative to unpaired training, particularly during the initial $50 \mathrm{~ms}$ of the CS. Neurons in MGm also showed greater activity during paired training relative to unpaired training that was sustained throughout the CS. Neurons in the P17-P19 group did not exhibit much learning-related activity and the SG even showed a decrease in activity with paired training.

\section{Discussion}

Developmental changes in medial auditory thalamic activity were evident during the unpaired pretraining session and following 

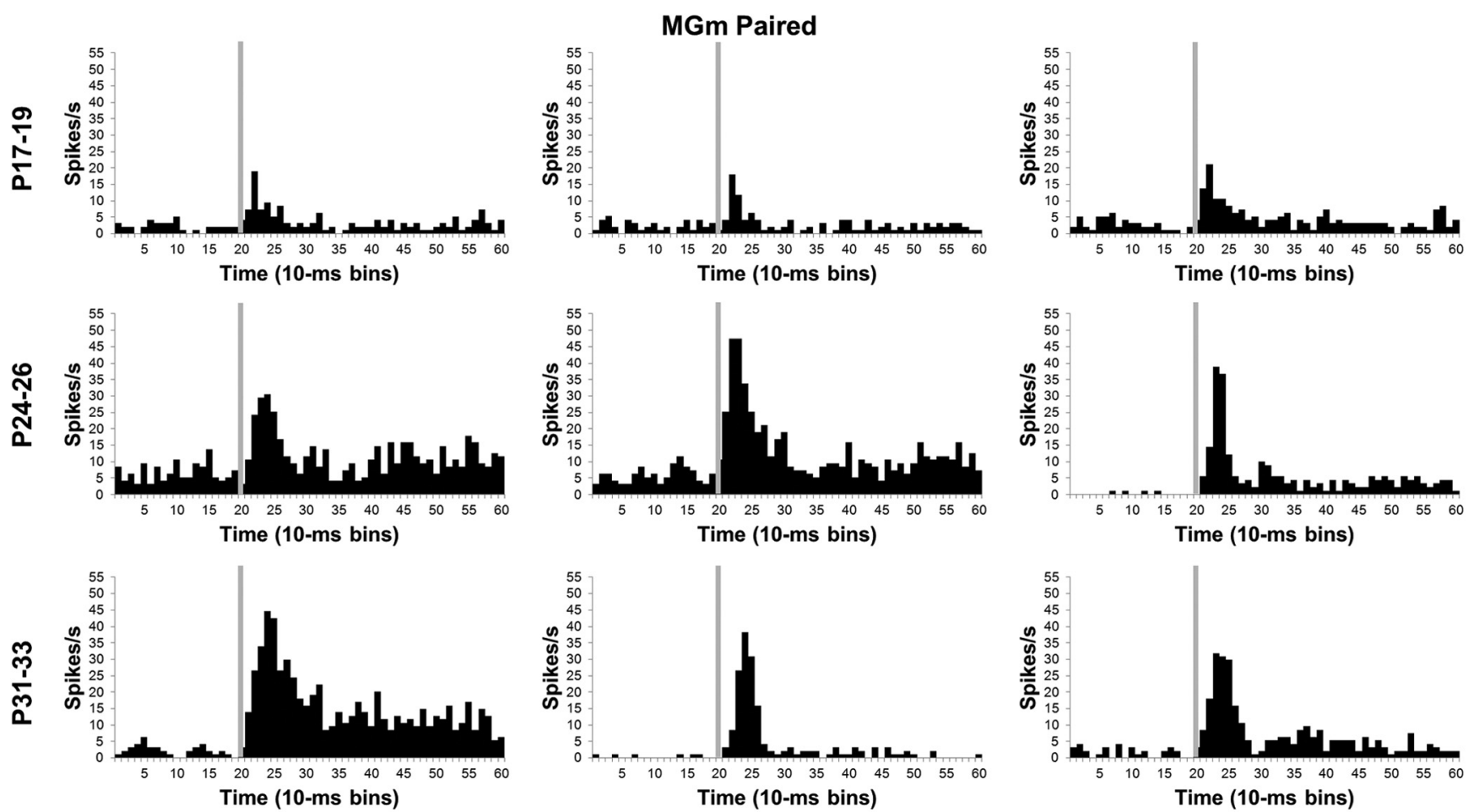

Figure 9. Histograms of single neuron activity in the MGm during presentations of the $400 \mathrm{~ms}$ tone conditioned stimulus in the last paired training session. Three examples are displayed for the groups trained on P17-P19 (top row), P24 -P26 (middle row), or P31-P33 (bottom row). Stimulus-elicited activity is greater in the neurons recorded from the older groups relative to the neurons recorded from the P17-P19 group during the first $100 \mathrm{~ms}$ of the tone stimulus and during the last $200 \mathrm{~ms}$ of the stimulus for some of the neurons. Stimulus onset is depicted by the vertical gray line.
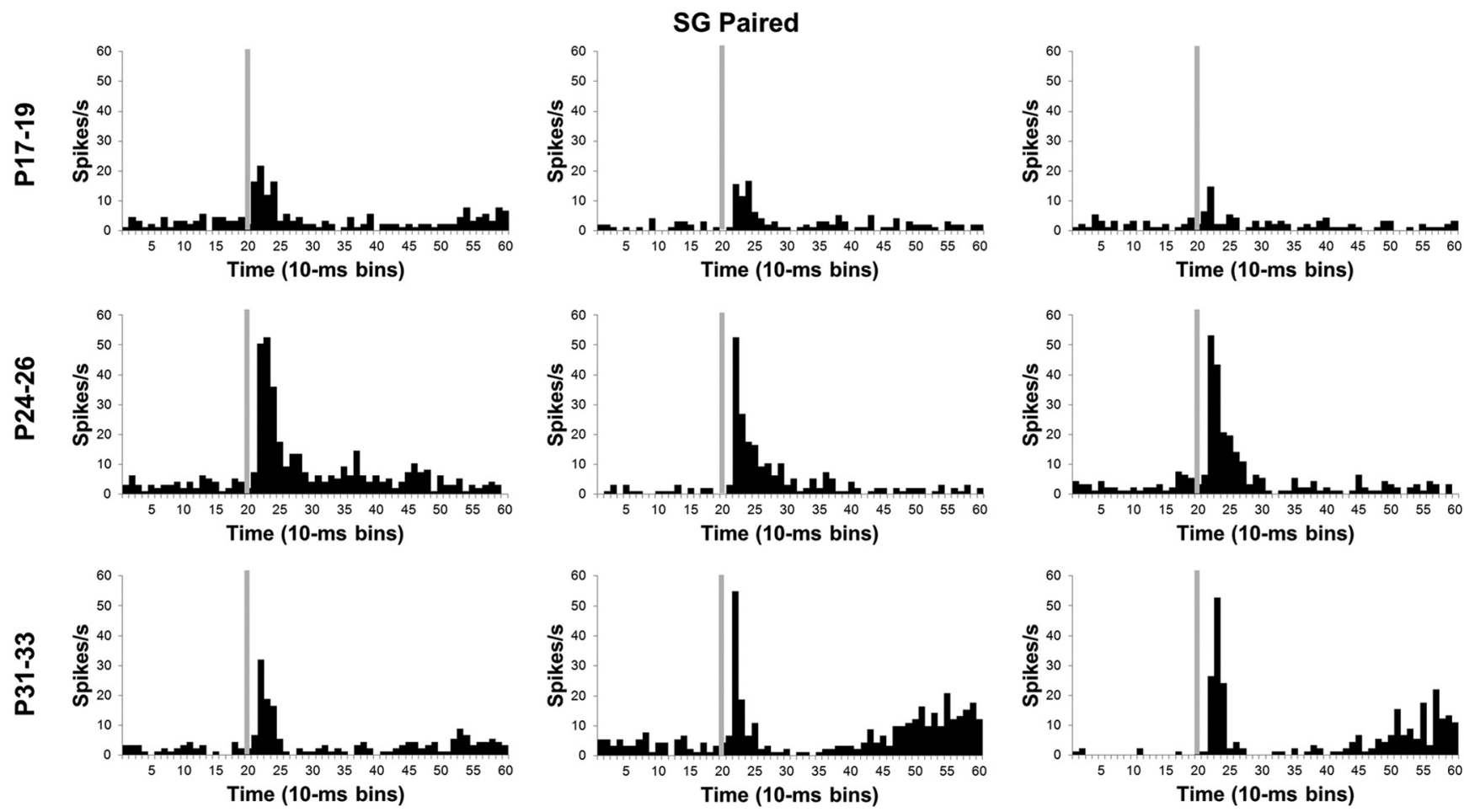

Figure 10. Histograms of single neuron activity in the $\mathrm{SG}$ nucleus during presentations of the $400 \mathrm{~ms}$ tone conditioned stimulus in the last paired training session. Three examples are displayed for the groups trained on P17-P19 (top row), P24-P26 (middle row), or P31-P33 (bottom row). Stimulus-elicited activity is greater in the neurons recorded from the older groups relative to the neurons recorded from the P17-P19 group during the first $100 \mathrm{~ms}$ of the tone stimulus and during the last $200 \mathrm{~ms}$ of the stimulus for some of the neurons. Stimulus onset is depicted by the vertical gray line. 
paired training. The proportion of neurons that responded to the tone CS did not differ as a function of postnatal age. Substantial developmental changes were seen, however, in the magnitude and duration of MAT activity during unpaired and paired training. During unpaired pretraining, MGm neurons showed an agerelated increase in activity during the CS, whereas SG neurons showed an agerelated decrease in activity during the CS. During paired training, both the MGm and SG neurons showed substantial developmental changes in the magnitude of CS-elicited activity and in learningrelated activity.

The SG and MGm showed a developmental difference in the initial sensory coding of the tone CS during unpaired pretraining. The developmental increase in the magnitude of tone-elicited activity in the MGm most likely reflects developmental changes in its synaptic input or excitability. Developmental changes in synaptic input could be in the axonal projection itself or in the development of upstream auditory nuclei. The higher magnitude of responding during unpaired pretraining in the SG of the P17P19 group relative to the older groups is more difficult to explain. The elevated responding in the P17-P19 group during unpaired pretraining is not likely due to sampling bias or some other confound because the neurons were recorded from multiple animals and the P24-P26 age group showed an intermediate pattern of responding between P17-P19 and P31P33. A possible mechanism for such elevated short-latency activity in the SG of the P17-P19 group during unpaired pretraining is a lack of local or modulatory inhibitory input to the SG. It is not clear, however, why there would be differential development of inhibition in the SG and MGm.

The observed developmental differences in tone-elicited short-latency activity in the SG and MGm during unpaired pretraining have important implications for the bottleneck hypothesis. The absence of a developmental change in the percentage of neurons responsive to the tone CS generally supports the bottleneck hypothesis. Moreover, if the SG and MGm make equal contributions to learning, then the developmental changes in these nuclei could summate, resulting in an overall more mature pattern of activity. This interpretation would also be consistent with the bottleneck hypothesis. If, however, the MGm contribution to learning is larger than the SG contribution, the bottleneck hypothesis is not sufficient because the thalamic changes would be upstream of the thalamo-pontine synaptic input. The relative contributions of the SG and MGm to cerebellar learning have not been determined yet. As a result, the current developmental pattern of thalamic activity cannot be precisely mapped onto developmental changes in learning.

In adult rats, there is a facilitation of short-latency $(<100 \mathrm{~ms})$ MAT activity to the CS with CS-US training relative to unpaired (No CR, gray line) are plotted.
$M G m$
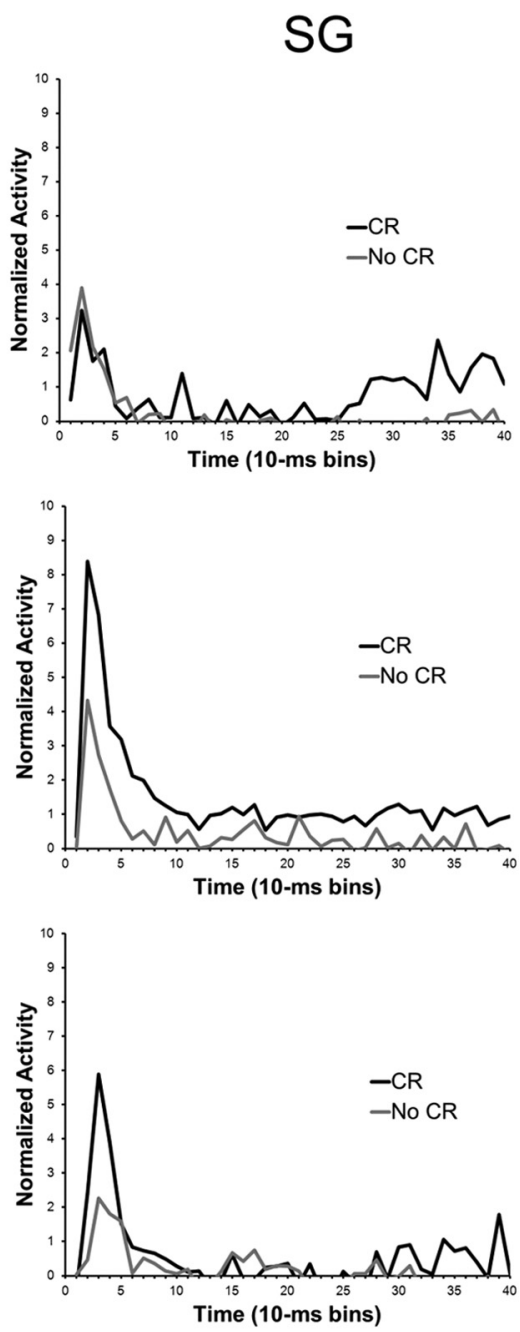

Figure 11. Plots of the average normalized activity from the population of neurons responsive to the $400 \mathrm{~ms}$ tone $\mathrm{CS}$ during the last paired training session in the MGm and SG nucleus from rat pups trained on P17-P19 (top row), P24-P26 (middle row), or P31-P33 (bottom row). Activity during trials with a conditioned response (CR, black line) and trials without a conditioned response

training (Halverson et al., 2010). Facilitated activity is also seen later in the CS period, which correlates with the amplitude and time course of the eyelid conditioned response (Halverson et al., 2010). The mechanism underlying the short-latency learningrelated facilitation is hypothesized to be input from the amygdala (Maren et al., 2001; Poremba and Gabriel, 2001; Halverson et al., 2010). The importance of this mechanism has been demonstrated in fear conditioning (Maren et al., 2001) and discriminative avoidance conditioning (Poremba and Gabriel, 2001) but not for EBC, although lesions of the amygdala impair acquisition of EBC (Neufeld and Mintz, 2001; Lee and Kim, 2004; Blankenship et al., 2005). The developmental change in short-latency learning-related activity in the current study might be related to developmental changes in amygdala input to the MAT or in learning-related activity within the amygdala itself. The latter possibility seems unlikely in that rat pups show robust auditory fear conditioning earlier than the youngest group in the current study (Hunt and Campbell, 1997; Stanton, 2000; Richardson and Hunt, 2010), but it has not been examined directly. Developmental changes in amygdala input to the MAT therefore seem more likely, but very little is known about how the amygdala influences the MAT. Evidence from a discriminative avoidance study in 
MGm
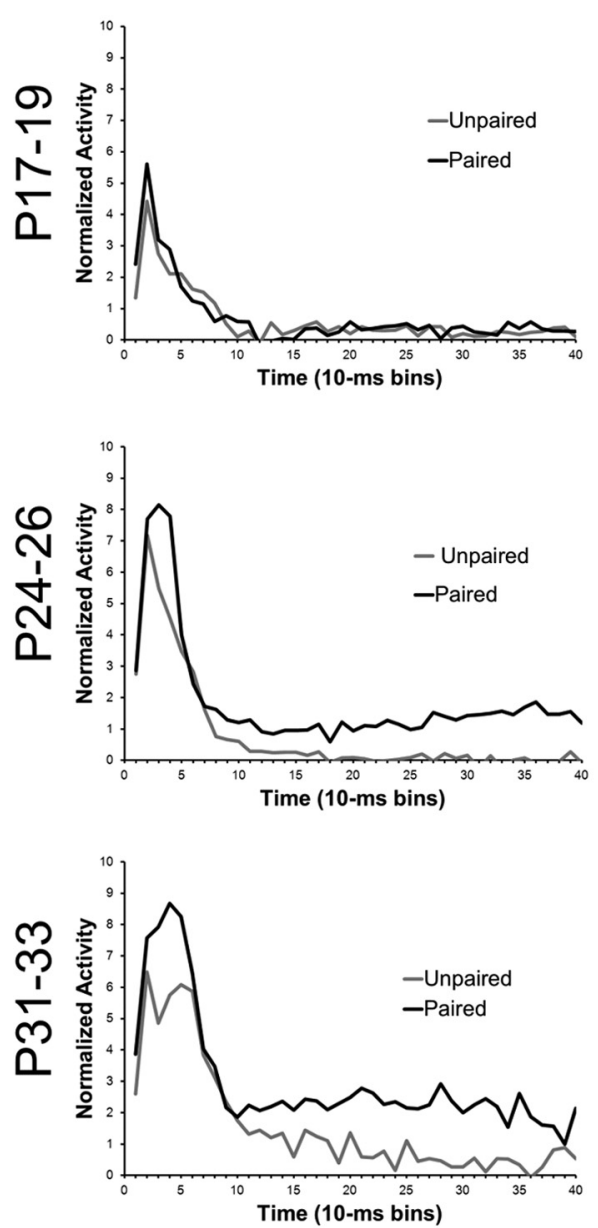

SG
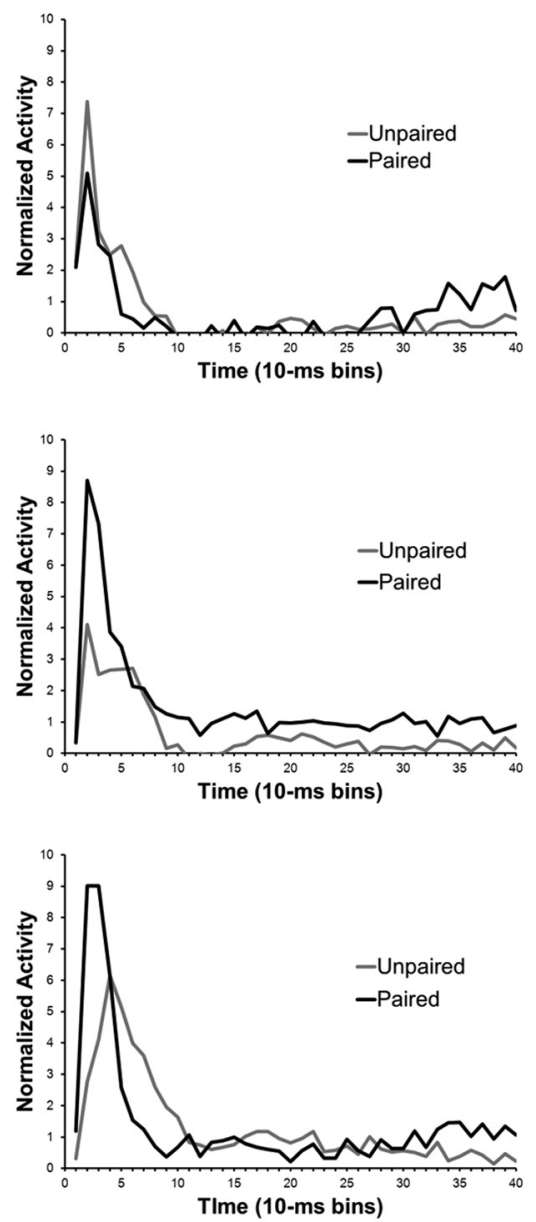

Figure 12. Plots of the average normalized activity from the population of neurons responsive to the $400 \mathrm{~ms}$ tone $C S$ during the unpaired pretraining session (Unpaired, gray line) and the last paired training session (Paired, black line) in the MGm and SG nucleus from rat pups trained on P17-P19 (top row), P24 -P26 (middle row), or P31-P33 (bottom row).

ulation of MAT activity is similar to that of fear-potentiated startle (Hunt and Campbell, 1997; Stanton, 2000; Richardson and Hunt, 2010). A corresponding developmental change in potentiation of the eyelid startle response was seen in the current study during EBC. Thus, amygdala modulation of the acoustic startle circuit (Walker and Davis, 2002) and the auditory CS pathway for EBC (Halverson et al., 2010) may develop in parallel.

In addition to the developmental changes in short-latency learning-related MAT activity, there were developmental changes in learning-related activity that occurs later in the CS period and correlates with the CR. This CR-related activity develops after cerebellar CR-related activity, both across training trials and within training trials, suggesting that it is driven by feedback from the cerebellum (Halverson et al., 2010). A monosynaptic projection from the cerebellar nuclei to the MAT was identified, which may be the substrate for the feedback (Halverson et al., 2010). Cerebellar feedback is hypothesized to produce a positive-feedback loop by facilitating MAT activity, which then facilitates pontine mossy fiber input back to the cerebellum. The hypothesized positivefeedback loop may increase the rate of cerebellar learning by facilitating induction of synaptic plasticity (Halverson et al., 2010). A similar positive-feedback loop has been identified for the cerebellar projections to the pontine and trigeminal nuclei (Clark and Lavond, 1996; Cartford et al., 1997; Clark et al., 1997; Bao et al., 2000). Developmental changes in CRrelated activity in the MAT are most likely

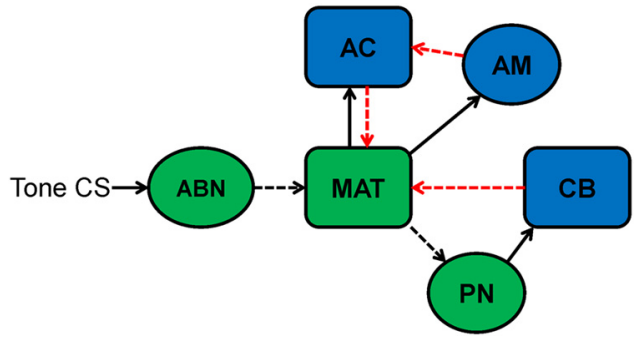

Figure 13. Diagram of the auditory CS neural pathway (green) and modulatory inputs to this pathway (blue). A tone CS initiates activity in auditory brainstem nuclei (ABN) that project to the medial auditory thalamus (MAT). The MAT then projects $C S$ input to the cerebellum (CB) through the pontine nuclei (PN). The cerebellum sends excitatory feedback to the MAT (red arrow), and the amygdala (AM) sends excitatory input to the MAT through the auditory cortex (AC) (red arrows). The dashed lines indicate possible sources of developmental change that directly or indirectly affect the ontogeny of MAT activity.

rabbits indicates that amygdala may project to the auditory cortex, which in turn projects back to the MAT (Duvel et al., 2001). It is possible that there are developmental changes in the amygdalo-cortical or cortico-thalamic projections mediating amygdala influences on learning-related activity in the MAT. The developmental time course of the hypothesized amygdala mod- caused by developmental changes in cerebellar learning. As sensory input to the pontine nuclei develops, synaptic plasticity in the cerebellum develops (Freeman, 2010). The development of cerebellar plasticity may then drive the development of CRrelated activity in the MAT. Developmental changes in the feedback mechanism itself are also possible. The ontogeny of cerebellar learning may therefore be influenced by developmental changes in sensory input to the pontine nuclei, synaptic plasticity in the cerebellum, and the positive-feedback loop between cerebellum and sensory thalamus (Fig. 13).

The current study demonstrated substantial ontogenetic changes in sensory- and learning-related activity in the MGm and SG. The magnitude of sensory-related activity during unpaired presentations of the auditory CS before training changed as a function of age. The developmental changes in sensory-related activity in the MAT are most likely driven by developmental changes in upstream auditory structures or their synaptic input to the thalamus (Fig. 13). Developmental changes in learningrelated activity within the MAT are most likely driven by developmental changes in amygdala and cerebellar input (Fig. 13). Amygdala input to the MAT, probably through the auditory cortex, may be developing in parallel with the development of learning. The positive-feedback loop between the cerebellum and 
MAT (Halverson et al., 2010) also appears to develop in parallel with learning (Fig. 13). The ontogeny of eyeblink conditioning is therefore influenced by developmental changes in MAT interactions with the pontine nuclei, amygdala, and cerebellum. Developmental changes in interactions between sensory and memory systems may underlie the ontogeny of other learning and memory processes as well. For example, the ontogeny of cued fear conditioning may be influenced by developmental changes in MAT and auditory cortical interactions with the amygdala. The developmental approach used in the current study and others provides a means for examining quantitative changes in the neural processes underlying learning in a manner that cannot be captured by lesion or inactivation studies in adult organisms.

\section{References}

Bao S, Chen L, Thompson RF (2000) Learning- and cerebellum-dependent neuronal activity in the lateral pontine nucleus. Behav Neurosci 114:254-261.

Blankenship MR, Huckfeldt R, Steinmetz JJ, Steinmetz JE (2005) The effects of amygdala lesions on hippocampal activity and classical eyeblink conditioning in rats. Brain Res 1035:120-130.

Campolattaro MM, Freeman JH (2008) Eyeblink conditioning in 12-dayold rats using pontine stimulation as the conditioned stimulus. Proc Natl Acad Sci U S A 105:8120-8123.

Campolattaro MM, Halverson HE, Freeman JH (2007) Medial auditory thalamic stimulation as a conditioned stimulus for eyeblink conditioning in rats. Learn Mem 14:152-159.

Campolattaro MM, Kashef A, Lee I, Freeman JH (2011) Neuronal correlates of cross-modal transfer in the cerebellum and pontine nuclei. J Neurosci 31:4051-4062.

Cartford MC, Gohl EB, Singson M, Lavond DG (1997) The effects of reversible inactivation of the red nucleus on learning-related and auditoryevoked unit activity in the pontine nuclei of classically conditioned rabbits. Learn Mem 3:519-531.

Clark RE, Lavond DG (1996) Neural unit activity in the trigeminal complex with interpositus or red nucleus inactivation during classical eyeblink conditioning. Behav Neurosci 110:13-21.

Clark RE, Gohl EB, Lavond DG (1997) The learning-related activity that develops in the pontine nuclei during classical eye-blink conditioning is dependent on the interpositus nucleus. Learn Mem 3:532-544.

Duvel AD, Smith DM, Talk A, Gabriel M (2001) Medial geniculate, amygdalar and cingulate cortical training-induced neuronal activity during discriminative avoidance learning in rabbits with auditory cortical lesions. J Neurosci 21:3271-3281.
Freeman JH (2010) Developmental neurobiology of cerebellar learning. In: Oxford handbook of developmental behavioral neuroscience (Blumberg MS, Freeman JH, Robinson SR, eds), pp 546-572. New York: Oxford UP.

Freeman JH, Campolattaro MM (2008) Ontogenetic change in the auditory conditioned stimulus pathway for eyeblink conditioning. Learn Mem 15:823-828.

Freeman JH, Steinmetz AB (2011) Neural circuitry and plasticity mechanisms underlying delay eyeblink conditioning. Learn Mem 18:666-677.

Freeman JH Jr, Muckler AS (2003) Developmental changes in eyeblink conditioning and neuronal activity in the pontine nuclei. Learn Mem 10:337-345.

Halverson HE, Freeman JH (2006) Medial auditory thalamic nuclei are necessary for eyeblink conditioning. Behav Neurosci 120:880-887.

Halverson HE, Lee I, Freeman JH (2010) Associative plasticity in the medial auditory thalamus and cerebellar interpositus nucleus during eyeblink conditioning. J Neurosci 30:8787-8796.

Hunt PS, Campbell BA (1997) Developmental dissociation of the components of conditioned fear. In: Learning, motivation, and cognition: the functional behaviorism of Robert C. Bolles (Bouton ME, Fanselow MS, eds), pp 53-74. Washington, DC: American Psychological Association.

Lee T, Kim JJ (2004) Differential effects of cerebellar, amygdalar, and hippocampal lesions on classical eyeblink conditioning in rats. J Neurosci 24:3242-3250.

Maren S, Yap SA, Goosens KA (2001) The amygdala is essential for the development of neuronal plasticity in the medial geniculate nucleus during auditory fear conditioning in rats. J Neurosci 21:RC135(1-6).

Neufeld M, Mintz M (2001) Involvement of the amygdala in classical conditioning of eyeblink response in the rat. Brain Res 889:112-117.

Poremba A, Gabriel M (2001) Amygdalar efferents initiate auditory thalamic discriminative training-induced neuronal activity. J Neurosci 21:270-278.

Richardson R, Hunt PS (2010) Ontogeny of fear conditioning. In: Oxford handbook of developmental behavioral neuroscience (Blumberg BM, Freeman JH, Robinson SR, eds), pp 527-545. New York: Oxford UP.

Stanton ME (2000) Multiple memory systems, development and conditioning. Behav Brain Res 110:25-37.

Stanton ME, Freeman JH Jr, Skelton RW (1992) Eyeblink conditioning in the developing rat. Behav Neurosci 106:657-665.

Swanson LW (2004) Brain maps: structure of the rat brain, Ed 3. London: Academic.

Thompson RF, Steinmetz JE (2009) The role of the cerebellum in classical conditioning of discrete behavioral responses. Neuroscience 162:732-755.

Walker DL, Davis M (2002) The role of amygdala glutamate receptors in fear learning, fear-potentiated startle, and extinction. Pharmacol Biochem Behav 71:379-392. 